\title{
DENSENESS OF OPERATORS WHOSE SECOND ADJOINTS ATTAIN THEIR NUMERICAL RADII
}

\author{
MARIA D. ACOSTA AND RAFAEL PAYA
}

(Communicated by John B. Conway)

\begin{abstract}
We show that for any Banach space the set of (bounded linear) operators whose second adjoints attain their numerical radii is norm-dense in the space of all operators. In particular, the numerical radius attaining operators on a reflexive space are dense.
\end{abstract}

B. Sims, paralleling the investigations by $\mathbf{J}$. Lindenstrauss on norm attaining operators, raised the question of the norm denseness of numerical radius attaining operators. To date, only partial answers to this question have been given. Berg and Sims [1] got an affirmative answer for uniformly convex spaces. The same result was shown by C. S. Cardassi [3] for uniformly smooth spaces. Cardassi has also shown that numerical radius attaining operators on $c_{0}, l_{1}$, $C(K)$ and $L_{1}(\mu)$ are dense $[4,5,6]$.

In this paper we obtain another sufficient condition for the denseness of numerical radius attaining operators, namely reflexivity of the space. In fact, we show that, given an operator $T$ on a Banach space $X$, there exists a compact operator $A$, with arbitrarily small norm, such that the second adjoint of $T+A$ attains its numerical radius. This result is analogous to the one obtained by Lindenstrauss for norm attaining operators [7, Theorem 1]. Our proof essentially consists of an adaptation of Lindenstrauss's proof in order to make it work for numerical radius attaining operators.

The dual space of a normed space $X$ will be denoted by $X^{*}$ and $B L(X)$ will be the normed space of bounded linear operators on $X$. The numerical radius of such an operator $T$ is defined by

$$
v(T)=\operatorname{Sup}\{|f(T(x))|:(x, f) \in \Pi(X)\}
$$

where $\Pi(X)=\left\{(x, f) \in X \times X^{*}: f(x)=\|f\|=\|x\|=1\right\}$ and we say that $T$ attains its numerical radius when there exists $\left(x_{0}, f_{0}\right)$ in $\Pi(X)$ such that

$$
\left|f_{0}\left(T\left(x_{0}\right)\right)\right|=v(T) .
$$

Received by the editors March 1, 1988.

1980 Mathematics Subject Classification (1985 Revision). Primary 47A12; Secondary 46B10.

Key words and phrases. Numerical radius, operators on Banach spaces, reflexive Banach spaces. 
We denote by $N R A(X)$ the set of operators on $X$ that attain their numerical radii.

Let $T^{*}$ denote the adjoint of $T$. It is well known that

$$
v\left(T^{*}\right)=v(T)
$$

[2, Corollary 9.6]. Let us write

$$
N R A_{0}(X)=\left\{T \in B L(X): T^{* *} \in N R A\left(X^{* *}\right)\right\} .
$$

It is clear that $N R A(X) \subset N R A_{0}(X)$ and both sets of operators are equal if $X$ is reflexive. We will prove that, for any Banach space $X, N R A_{0}(X)$ is norm-dense in $B L(X)$. We need the following characterization of operators in $N R A_{0}(X)$, which is analogous to the one given in [7, Lemma 1] for norm attaining operators.

Lemma 1. Let $X$ be a normed space and $S \in B L(X)$. Assume that there are sequences $\left\{x_{n}\right\}$ in $X,\left\{f_{n}\right\}$ in $X^{*}$ and $\left\{\delta_{n}\right\},\left\{\varepsilon_{n}\right\}$ in $\mathbf{R}^{+}$, satisfying

(a) $\left\|f_{n}\right\|=\left\|x_{n}\right\|=1$,

(b) $\left\{\delta_{n}\right\} \rightarrow 0,\left\{\varepsilon_{n} / \delta_{n}\right\} \rightarrow 0$ and

(c) $1+\delta_{n} v(S) \leq\left|f_{n}\left(x_{n+k}\right)\right|+\delta_{n}\left|f_{n}\left(S\left(x_{n+k}\right)\right)\right|+\varepsilon_{n}$ for all positive integers $n$ and $k$.

Then $S \in N R A_{0}(X)$.

Proof. Let $F$ and $\Psi$ be cluster points of the sequences $\left\{x_{n}\right\}$ and $\left\{f_{n}\right\}$ in the $w^{*}$-topologies of $X^{* *}$ and $X^{* * *}$, respectively. From (c), by keeping $n$ fixed and varying $k$, we deduce that

$$
1+\delta_{n} v(S) \leq\left|F\left(f_{n}\right)\right|+\delta_{n}\left|F\left(S^{*}\left(f_{n}\right)\right)\right|+\varepsilon_{n}
$$

holds for all $n$, so

$$
1 \leq|\Psi(F)| \leq 1 \quad \text { and } \quad\left(F, \frac{\Psi}{\Psi(F)}\right) \in \Pi\left(X^{* *}\right) .
$$

Since $\left|F\left(f_{n}\right)\right| \leq 1$, we also get from $(*)$ that

$$
v(S) \leq\left|F\left(S^{*}\left(f_{n}\right)\right)\right|+\frac{\varepsilon_{n}}{\delta_{n}}
$$

for all $n$, so

$$
v(S) \leq\left|\Psi\left(S^{* *}(F)\right)\right|
$$

and $S^{* *}$ attains its numerical radius.

Remark 2. The sufficient condition given by the above lemma for an operator $S$ to belong to $N R A_{0}(X)$ is also necessary. It is not difficult to show that if $S \in N R A_{0}(X)$ and $\left\{\delta_{n}\right\},\left\{\varepsilon_{n}\right\}$ are sequences of positive real numbers satisfying the condition (b) of the lemma, then sequences $\left\{x_{n}\right\}$ and $\left\{f_{n}\right\}$ can be chosen so as to satisfy (a) and (c).

Our next technical lemma shows the route we will follow in order to construct operators satisfying the assumptions of Lemma 1. 
Lemma 3. Let $X$ be a normed space and $T \in B L(X)$. Given three positive real constants $\alpha, \delta$ and $\rho$, let $(x, f) \in \Pi(X)$ be such that

$$
|f(T(x))|>v(T)-\alpha .
$$

Let us define a new operator $T^{\prime}$ by

$$
T^{\prime}(z)=T(z)+\lambda \delta f(z) x+\delta^{2} f(T(z)) x \quad(z \in X)
$$

where $\lambda$ is a scalar such that $|\lambda|=1$ and $f(T(x))=\lambda|f(T(x))|$. If $(y, g) \in$ $\Pi(X)$ is such that $\left|g\left(T^{\prime}(y)\right)\right|>v\left(T^{\prime}\right)-\rho$, then

$$
1+\delta v(T) \leq|f(y)|+\delta|f(T(y))|+\frac{\rho}{\delta}+\frac{\alpha\left(1+\delta^{2}\right)}{\delta} .
$$

Proof. We clearly have

$$
\begin{aligned}
v\left(T^{\prime}\right) & \geq\left|f\left(T^{\prime}(x)\right)\right|=\left|f(T(x))+\lambda \delta+\delta^{2} f(T(x))\right| \\
& =\delta+\left(\delta^{2}+1\right)|f(T(x))| \geq \delta+\left(\delta^{2}+1\right)(v(T)-\alpha) .
\end{aligned}
$$

On the other hand,

$$
\begin{aligned}
v\left(T^{\prime}\right) & \leq\left|g\left(T^{\prime}(y)\right)\right|+\rho \leq|g(T(y))|+\delta|f(y)|+\delta^{2}|f(T(y))|+\rho \\
& \leq v(T)+\delta|f(y)|+\delta^{2}|f(T(y))|+\rho .
\end{aligned}
$$

The desired inequality follows by combining the above inequalities and dividing by $\delta$.

Theorem 4. For every Banach space $X, N R A_{0}(X)$ is norm-dense in $B L(X)$. Moreover, every operator on $X$ may be perturbed by a compact operator of arbitrarily small norm, to obtain an operator in $N R A_{0}(X)$.

Proof. Let $T \in B L(X)$ with $\|T\|=1$ and $0<\varepsilon<\frac{1}{2}$ be given. We choose two decreasing sequences of positive numbers, $\left\{\delta_{n}\right\}$ and $\left\{\alpha_{n}\right\}$, satisfying

$$
\sum_{j=1}^{\infty}\left(\delta_{j}+2 \delta_{j}^{2}\right)<\varepsilon ; \quad \lim _{n \rightarrow \infty} \frac{1}{\delta_{n}^{2}} \sum_{j=n+1}^{\infty}\left(\delta_{j}+2 \delta_{j}^{2}\right)=0 ; \quad\left\{\frac{\alpha_{n}}{\delta_{n}^{2}}\right\} \rightarrow 0 .
$$

(We could choose, for example, $\delta_{n}=\varepsilon / 3 \cdot 2^{n !}$ and $\alpha_{n}=\delta_{n}^{3}$.) Next we inductively construct sequences $\left\{T_{n}\right\}$ in $B L(X)$ and $\left\{\left(x_{n}, f_{n}\right)\right\}$ in $\Pi(X)$ satisfying

$$
\begin{gathered}
T_{1}=T, \\
\left|f_{n}\left(T_{n}\left(x_{n}\right)\right)\right|>v\left(T_{n}\right)-\alpha_{n}, \\
T_{n+1}(x)=T_{n}(x)+\delta_{n} \lambda_{n} f_{n}(x) x_{n}+\delta_{n}^{2} f_{n}\left(T_{n}(x)\right) x_{n} \quad(x \in X),
\end{gathered}
$$

where $\left|\lambda_{n}\right|=1$ and $f_{n}\left(T_{n}\left(x_{n}\right)\right)=\lambda_{n}\left|f_{n}\left(T_{n}\left(x_{n}\right)\right)\right|$.

One can easily verify by induction that

$$
\left\|T_{n+1}\right\| \leq 1+\sum_{j=1}^{n}\left(\delta_{j}+2 \delta_{j}^{2}\right) \leq 2, \text { for all } n
$$


It follows that

$$
\left\|T_{n+k}-T_{n}\right\| \leq \sum_{j=n}^{n+k-1}\left(\delta_{j}+2 \delta_{j}^{2}\right), \quad \text { for all } n, k .
$$

In view of (6) and (1), the sequence $\left\{T_{n}\right\}$ converges in norm to an operator $S$ in $B L(X)$ which satisfies

$$
\left\|S-T_{n}\right\| \leq \sum_{j=n}^{\infty}\left(\delta_{j}+2 \delta_{j}^{2}\right), \quad \text { for all } n,
$$

and in particular $\|S-T\|<\varepsilon$.

We claim that $S$ satisfies the requirements of Lemma 1. To prove this, let $n$ and $k$ be fixed natural numbers and let us note that

$$
\begin{aligned}
\left|f_{n+k}\left(T_{n+1}\left(x_{n+k}\right)\right)\right| & \geq\left|f_{n+k}\left(T_{n+k}\left(x_{n+k}\right)\right)\right|-\left\|T_{n+k}-T_{n+1}\right\| \\
& \geq v\left(T_{n+k}\right)-\alpha_{n+k}-\left\|T_{n+k}-T_{n+1}\right\| \\
& \geq v\left(T_{n+1}\right)-\alpha_{n+k}-2\left\|T_{n+k}-T_{n+1}\right\| \\
& \geq v\left(T_{n+1}\right)-\alpha_{n+k}-2 \sum_{j=n+1}^{\infty}\left(\delta_{j}+2 \delta_{j}^{2}\right),
\end{aligned}
$$

where we have used (3) and (6).

Now we can apply Lemma 3, with $T_{n}$ playing the role of $T, \alpha=\alpha_{n}, \delta=\delta_{n}$, $\rho=\alpha_{n+k}+2 \sum_{j=n+1}^{\infty}\left(\delta_{j}+2 \delta_{j}^{2}\right),(x, f)=\left(x_{n}, f_{n}\right)$ and $(y, g)=\left(x_{n+k}, f_{n+k}\right)$, so that the operator $T^{\prime}$ defined in the statement of Lemma 3 agrees with $T_{n+1}$ and the lemma gives

$$
\begin{aligned}
1+\delta_{n} v\left(T_{n}\right) \leq & \left|f_{n}\left(x_{n+k}\right)\right|+\delta_{n}\left|f_{n}\left(T_{n}\left(x_{n+k}\right)\right)\right| \\
& +\frac{1}{\delta_{n}}\left[\alpha_{n+k}+2 \sum_{j=n+1}^{\infty}\left(\delta_{j}+2 \delta_{j}^{2}\right)+\alpha_{n}\left(1+\delta_{n}^{2}\right)\right] \\
\leq & \left|f_{n}\left(x_{n+k}\right)\right|+\delta_{n}\left|f_{n}\left(T_{n}\left(x_{n+k}\right)\right)\right| \\
& +\frac{1}{\delta_{n}}\left[2 \sum_{j=n+1}^{\infty}\left(\delta_{j}+2 \delta_{j}^{2}\right)+\alpha_{n}\left(2+\delta_{n}^{2}\right)\right],
\end{aligned}
$$

where, for the last inequality, we used the fact that $\left\{\alpha_{n}\right\}$ is a decreasing sequence.

Now we need to put $S$ in place of $T_{n}$ in the above inequality. To dominate the errors we use the estimate of $\left\|S-T_{n}\right\|$ given by (7) and we obtain

$$
1+\delta_{n} v(S) \leq\left|f_{n}\left(x_{n+k}\right)\right|+\delta_{n}\left|f_{n}\left(S\left(x_{n+k}\right)\right)\right|+\varepsilon_{n}
$$

where

$$
\varepsilon_{n}=\frac{1}{\delta_{n}}\left[2 \sum_{j=n+1}^{\infty}\left(\delta_{j}+2 \delta_{j}^{2}\right)+\alpha_{n}\left(2+\delta_{n}^{2}\right)\right]+2 \delta_{n} \sum_{j=n}^{\infty}\left(\delta_{j}+2 \delta_{j}^{2}\right)
$$


By (1) the sequences $\left\{\delta_{n}\right\}$ and $\left\{\varepsilon_{n}\right\}$ satisfy the conditions in Lemma 1 , hence $S \in N R A_{0}(X)$.

Finally, let us remark that $S-T$ is the sum of a norm-convergent series whose terms are rank-one operators, so $S-T$ is compact.

Corollary 5. If $X$ is a reflexive Banach space, then $N R A(X)$ is norm-dense in $B L(X)$.

\section{REFERENCES}

1. I. D. Berg and B. Sims, Denseness of numerical radius attaining operators, J. Austral. Math. Soc. Ser. A 36 (1984), 130-133.

2. F. F. Bonsall and J. Duncan, Numerical ranges of operators on normed spaces and of elements of normed algebras, London Math. Soc. Lecture Note Series, No. 2, Cambridge Univ. Press, 1971.

3. C. S. Cardassi, Density of numerical radius attaining operators on some reflexive spaces, Bull. Austral. Math. Soc. 31 (1985), 1-3.

4. ___ Numerical radius attaining operators, Lecture Notes in Math., vol. 1166, Springer-Verlag, Berlin and New York, 1985, pp. 11-14.

5. __ Numerical radius attaining operators on $C(K)$, Proc. Amer. Math. Soc. 95 (1985), 537-543.

6. __ Numerical radius attaining operators on $L_{1}(\mu)$, Preprint, University of São Paulo.

7. J. Lindenstrauss, On operators which attain their norm, Israel J. Math. 1 (1963), 139-148.

Departamento de Análysis Matemático, facultad de Ciencias, Universidad de Granada, 18071 Granada, Spain 